\title{
Efeitos de extratos alcoólicos de plantas sobre o caruncho do feijão vigna (Callosobruchus maculatus)
}

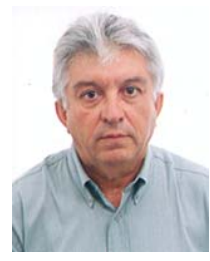

Francisco de A. C. Almeida1, Silvana A. de Almeida², Nilene R. dos Santos², Josivanda P. Gomes ${ }^{1} \&$ Maria E. R. Araújo ${ }^{2}$

\begin{abstract}
${ }^{1}$ DEAg/UFCG. Av. Aprígio Veloso, 882, Fone: (83) 3310-1185. E-mail: diassis@deag.ufcg.edu.br, e josi@deag.ufcg.edu.br
2 Pós-Graduanda. E-mail: silvanalves@yahoo.com.br; nileners@yahoo.com.br; ele.sandra@bol.com.br
\end{abstract}

Protocolo 196 - 15/12/2003 - Aprovado em 13/4/2005

\begin{abstract}
Resumo: Através de três métodos, extratos vegetais foram aplicados, ao Callosobruchus maculatus na fase adulta, inoculados ou não em uma massa de sementes, e na fase imatura (ovo) com o objetivo de se controlar esta praga do feijão armazenado. Utilizaram-se flores, folhas, frutos e caule secos de oito espécies vegetais na obtenção dos extratos, em percolador, com solvente álcool etílico (70\%). O delineamento experimental utilizado foi o inteiramente ao acaso, com os tratamentos distribuídos em esquema fatorial, cujos fatores quantitativos foram revelados pela regressão na análise de variância. Mediante os resultados obtidos, concluiu-se que a mortalidade dos insetos está relacionada com o tipo de extrato, os métodos de aplicação e com a dosagem aplicada, sendo os extratos de Callopogonium caeruleum e Piper nigrum os mais eficientes no controle do caruncho de feijão.
\end{abstract}

Palavras-chave: insetos-praga, mortalidade, sementes

\section{Effect of alcoholic extract of plants on weevil of cowpea (Callosobruchus maculatus)}

\begin{abstract}
Vegetable extracts were applied, through three methods, to the Callosobruchus maculatus in the adult phase, inoculated or not in a mass of seeds, in the immature phase (egg) with the objective of controlling this pest of the stored beans. Dry flowers, leaves, fruits and dry stems of eight vegetable species were used to obtain the extracts in an extractor, with ethyl alcohol $(70 \%)$. A completely randomized statistical design was used with the treatments distributed in a factorial scheme, the quantitative factors were analysed by the regression in the variance analysis. From the results obtained, it was concluded that the mortality of the insects is related to the extract type, the application methods and the applied dose, being the extracts of Callopogonium caeruleum and Piper nigrum the most efficient in the control of the weevil of cowpea.
\end{abstract}

Key words: pest-insects, mortality, seeds

\section{INTRODUÇÃO}

O caruncho, Callosobruchus maculatus (Coleoptera: Bruchidae) considerado principal inseto-praga do feijão armazenado, tem seus danos decorrentes da penetração e alimentação das larvas no interior das sementes, provocando perda de peso, redução do poder germinativo, do valor nutritivo das sementes e grãos, e do grau de higiene do produto, pela presença de excrementos, ovos e insetos.

Seu controle, assim como dos demais insetos-praga associados aos grãos armazenados, tem sido realizado em larga escala, por meio de produtos químicos fumigantes. Relatos sobre o surgimento de mecanismos de resistência a tratamentos químicos em várias espécies de insetos-praga são cada vez mais constantes (Martinazzo et al., 2000). As pesquisas atuais e o aumento no conhecimento dos prejuízos advindos do uso indiscriminado desses produtos vêm encorajando estudos relacionados a novas técnicas de controle relacionado a essas pragas, dentre elas o uso de inseticidas de origem vegetal. Tais inseticidas, também chamados inseticidas botânicos, são alvo constante de inúmeras pesquisas nas quais se apresentam resultados satisfatórios, além de serem de fácil utilização e obtenção, de baixo custo e, ainda, minimizarem os problemas apresentados pelos produtos químicos, constituindo-se em um importante método de controle a ser adotado principalmente pelos pequenos agricultores (Tavares, 2002).

A utilização de plantas inseticidas como método alternativo de controle de pragas, não é uma técnica recente e seu uso é 
comum, sobretudo em países tropicais, antes mesmo do advento dos inseticidas sintéticos. O ressurgimento das pesquisas com plantas inseticidas ocorreu em razão da necessidade de novos compostos biorracionais que controlem as pragas sem provocar problemas ao homem nem ao ambiente. O emprego de plantas inseticidas tem ganhado importância em especial no segmento dos alimentos orgânicos, cujo cultivo e consumo vêm crescendo rapidamente em todo o mundo, nos últimos anos (Vendramim, 2000).

A utilização de plantas com atividade inseticida no controle de pragas de armazenamento se deve, sobretudo ao surgimento da resistência dos insetos aos inseticidas organossintéticos, à contaminação causada por elas, à presença de resíduos químicos tóxicos nos alimentos e à intoxicação dos operários aplicadores de inseticidas (Hernández \& Vendramim, 1997).

As pesquisas com plantas inseticidas são realizadas, basicamente, com o objetivo de se descobrir moléculas com atividade contra insetos que permitam a síntese de novos produtos inseticidas e a obtenção de inseticidas naturais para o uso direto no controle de insetos-praga (Vendramim, 2000). Sobre o tema, Miyakado et al. (1989); Flores et al. (1993); Boff \& Almeida (1996); Goldfarb (1997); Lale \& Abdulrahman(1999); Oliveira (1998) e Kéita et al. (2001), referenciam estudos com extratos de origem vegetal e seus efeitos sobre diversas espécies de insetos-praga de sementes e grãos armazenados.

Assim, como o avanço nas pesquisas relacionadas aos inseticidas de origem vegetal possibilita perspectivas para sua utilização no controle dos insetos praga de grãos e sementes armazenados, desenvolveu-se este trabalho com o propósito de se avaliar, em condições de laboratório, a eficácia de extratos vegetais no controle ao Callosobruchus maculatus, nas fases adulta e imatura (ovo).

\section{MATERIAL E MÉTODOS}

O trabalho foi desenvolvido no Laboratório de Armazenamento e Pré-Processamento de Produtos Agrícolas (LAPPA) da Unidade Acadêmica de Engenharia Agrícola da Universidade Federal de Campina Grande (UFCG), Campina Grande, PB.

Os insetos foram multiplicados em laboratório sob condições ambientais, dentro de recipientes de vidro transparente, contendo $0,5 \mathrm{~kg}$ de feijão, previamente expurgado; os extratos foram obtidos a partir de oito espécies vegetais (Tabela 1), citadas na literatura especializada como possuidores de princípios ativos no controle de insetos-praga de grãos e sementes armazenadas ou, ainda, por serem popularmente detentoras desses princípios, sendo estes submetidos a uma secagem em estufa a temperatura constante de $40{ }^{\circ} \mathrm{C}$, durante $48 \mathrm{~h}$; posteriormente, o material seco foi triturado em moído de facas elétrico, até a formação de massa fina (pó).

\section{Obtenção dos extratos}

A massa obtida depois da trituração, conforme já descrito, foi levada ao percolador de extração, na qual o solvente extrator (álcool etílico a 70\%) passava retirando-se os princípios ativos, conforme metodologia de Prista \& Alves (1996).
Tabela 1. Espécies botânicas utilizadas na obtenção dos extratos vegetais

\begin{tabular}{lll}
\hline Nome Científico & Nome Vulgar & Parte Utilizada \\
\hline Anona squamosa & Pinha & Semente \\
Anthemis nobilis & Camomila romana & Flores \\
Azadiracta indica & Nim & Folhas \\
Camellia sinensis & Chá preto & Flores \\
Croton tiglium & Cróton & Folha \\
Calopogonium caeruleum & Cipó - Timbó & Caule \\
Mentha piperita & Hortelã pimenta & Folhas \\
Piper nigrum & Pimenta do reino & Fruto
\end{tabular}

\section{Mortalidade dos Callosobruchus maculatus}

A mortalidade dos insetos adultos se deu com base no princípio tóxico dos extratos levados na forma de vapor (Almeida et al., 1999), onde estes se encontravam e inalavam o extrato pela traquéia e demais vias respiratórias. Utilizou-se um compressor adaptado que liberava o ar ao passar por um recipiente que, por sua vez, levava o extrato nele contido, na forma de vapor, diretamente aos recipientes em que se encontravam os insetos; estes mediam $15 \mathrm{~cm}$ de altura por 10 $\mathrm{cm}$ de diâmetro, eram de plástico, suas tampas eram perfuradas em dois locais, uma para a entrada do vapor (extrato) e a outra para a movimentação do oxigênio durante a aplicação. Utilizaram-se 50 insetos adultos com três dias de idade em cada recipiente e os tratamentos constaram de 4 repetições para cada dose testada $(3,6,9$ e $12 \mathrm{~mL})$. A avaliação foi efetuada depois de $48 \mathrm{~h}$ da aplicação dos extratos, considerando-se vivos todos os insetos que moviam qualquer parte do corpo, mesmo aqueles que permaneciam imóveis e só se moviam lentamente, quando incomodados com estímulos físicos.

\section{Métodos de aplicação dos extratos}

Avaliaram-se os 4 extratos que se mostraram mais eficazes sobre a mortalidade do C. maculatus adulto, a dosagem $(3 \mathrm{~mL})$ eleita pela eficácia estatística e também o custo de aplicação, decorrente dos resultados revelados na etapa anterior. O experimento foi realizado em silos metálicos de tamanho reduzido ( $12 \mathrm{~cm}$ de altura por $6 \mathrm{~cm}$ de diâmetro) com capacidade para $0,5 \mathrm{~kg}$ de feijão variedade "rabo de tatu", previamente expurgada com fosfina; posteriormente, os extratos, na forma de vapor, foram aplicados diretamente na massa onde se encontravam 50 insetos, com ou sem o auxílio de um tubo de PVC perfurado em toda a sua extensão, que havia sido introduzido no centro da massa de sementes para permitir uma difusão melhor dos gases. Após três dias da aplicação dos extratos, os adultos foram retirados e contados os sobreviventes, os quais foram descartados, avaliando-se a percentagem de infestação inicial (ovos); depois de 30 dias da aplicação dos extratos foi avaliada a percentagem de infestação final (orifícios de emergência dos adultos). Na aplicação direta, as sementes, previamente expurgadas, foram tratadas e postas sobre papel monofoliado para secagem natural, durante $24 \mathrm{~h}$; após isto, foram levados aos silos metálicos e inoculados 50 insetos para oviposição. Passados 3 dias da introdução dos insetos, os adultos vivos foram contados e descartados, avaliando-se a percentagem de infestação inicial (ovos) e final (orifícios de emergência dos adultos), após 30 dias. 


\section{Inviabilidade de ovos de Callosobruchus maculatus}

Em $0,5 \mathrm{~kg}$ de feijão previamente expurgado, introduziram-se 50 insetos adultos para oviposição; depois de $72 \mathrm{~h}$, todos os insetos foram retirados e computados o percentual de infestação inicial (ovos). As sementes ovipositadas foram tratadas com 3 e $6 \mathrm{~mL}$ dos respectivos extratos de C. caeruleum e $P$. nigrum e depois de 20 dias do tratamento e posterior a cada cinco dias, até 45 dias, foi avaliado o índice de emergência dos adultos.

\section{Cálculo de infestação e perda de peso}

A percentagem de infestação e perda de peso foram calculadas a partir de $100 \mathrm{~g}$ da amostra, utilizando-se as seguintes fórmulas, descritas por Almeida \& Villamil (2000)

$$
P I=\frac{D}{D+I} \times 100
$$

em que:

PI - porcentagem de infestação

D - número de sementes danificadas

I - número de sementes íntegras

$$
\mathrm{PP}=\frac{\mathrm{I}-\mathrm{D}}{\mathrm{I}} \times 100
$$

em que:

PP - porcentagem de perda de peso

$\mathrm{D}$ - peso do número de sementes danificadas

I - peso do número de sementes íntegras

\section{Análise dos resultados}

Os resultados foram analisados através do Programa Computacional Assistat versão 6.5 (Silva \& Azevêdo, 2002, 2003), utilizando-se o delineamento DIC (Delineamento Inteiramente Casualisado), disposto em esquema fatorial com 4 repetições. A comparação entre as médias foi realizada pelo teste de Tukey, a 1 e 5\% de probabilidade e, para os fatores quantitativos (dosagens) estudou-se a regressão na análise de variância.

\section{RESULTADOS E DISCUSSÃO}

\section{Mortalidade dos Callosobruchus maculatus}

A análise de variância (Tabela 2) revelou efeito altamente significativo para extrato (E), dose (D) e interação E x D. Por se tratar do fator D quantitativo, procedeu-se ao desdobramento da interação, através da regressão polinomial, na qual se obteve efeito linear significativo para todas as variáveis, exceto para Azadiracta indica, e quadrático apenas para o C. tiglium.

Mediante os resultados relativos à quantidade (dose) dos extratos que foram aplicados na forma de vapor ao $C$. maculatus adulto (Figura 1) tem-se uma relação positiva, isto é, a eficiência progride com o aumento da quantidade do extrato aplicado ao caruncho adulto, comportamento este observado pelos polinômios testados para fins do estudo, cuja regressão na análise de variância mostrou alta significância e coeficiente de determinação $\left(\mathrm{R}^{2}\right)$ acima de $96 \%$, indicando que as equações
Tabela 2. Quadrado médio da variância e da regressão para a mortalidade do caruncho adulto do feijão vigna (Callosobruchus maculatus) após $48 \mathrm{~h}$ da aplicação dos

\begin{tabular}{|c|c|c|c|c|}
\hline Fonte de Variação & G.L. & Q.M. & $\mathrm{R}^{2}$ & $\mathrm{CV}(\%)$ \\
\hline Extrato (E) & 7 & $3825,9284^{* *}$ & & \\
\hline Dosagem (D) & 3 & $520,6250^{* *}$ & & \\
\hline$E \times D$ & 21 & $46,3273^{* *}$ & & \\
\hline Resíduo & 96 & 8,05208 & & 3,17 \\
\hline \multicolumn{5}{|c|}{ Camellia sinensis } \\
\hline Linear & 1 & $460,80^{* *}$ & 0,97 & 3,59 \\
\hline \multicolumn{5}{|c|}{ Anthemis nobilis } \\
\hline Linear & 1 & $432,4500^{* *}$ & 0,99 & 2,39 \\
\hline \multicolumn{5}{|c|}{ Mentha piperita } \\
\hline Linear & 1 & $460,8000^{* *}$ & 0,97 & 3,59 \\
\hline \multicolumn{5}{|c|}{ Cróton tiglium } \\
\hline Quadrática & 1 & $100,00^{*}$ & 0,99 & ,68 \\
\hline \multicolumn{5}{|c|}{ Annona scamosa } \\
\hline Linear & 1 & $33,800^{*}$ & 0,97 & 2,14 \\
\hline \multicolumn{5}{|c|}{ Piper nigrum } \\
\hline Linear & 1 & $28,800^{* *}$ & 0,96 & 0,93 \\
\hline \multicolumn{5}{|c|}{ Calopogonium caeruleum } \\
\hline Linear & 1 & $12,800^{*}$ & 0,98 & 1,49 \\
\hline \multicolumn{5}{|c|}{ Azadiracta indica } \\
\hline Linear & 1 & $5,00^{\mathrm{ns}}$ & & 1,08 \\
\hline Quadrática & 1 & $1,00^{\mathrm{ns}}$ & & \\
\hline
\end{tabular}
extratos

podem ser empregadas para simular os intervalos dos dados obtidos experimentalmente. Sobre o tema, Kéita et al. (2001) estudaram a eficiência dos extratos $O$. basilicum e $O$. gratissimum no controle do C. maculatus e concluíram que a eficácia aumenta com o aumento das dosagens utilizadas dos extratos.

Para os extratos, (Tabela 3), os efeitos foram distintos, tendo a $A$. squamosa, o P. nigrum, o C. caeruleum e $A$. indica, se mostrado os mais eficientes no controle dessa praga do feijão armazenado, controlando a presença da mesma acima de $95 \%$; já na menor dosagem ( $3 \mathrm{~mL})$; no entanto, em termos de valor absoluto $(100 \%)$ a $A$. indica foi o mais agressivo, tendo matado todos os C. maculatus a partir da dosagem de $9 \mathrm{~mL}$, comportamento registrado para o C. caeruleum quando a dosagem aplicada foi de $12 \mathrm{~mL}$. Esses quatro extratos superaram estatisticamente os demais extratos, quando levados ao inseto praga do feijão armazenado nas quantidades de 3, 6 e $9 \mathrm{~mL}$ e, com exceção do extrato de $C$. sinensis, todos os extratos mataram igualmente, conforme os resultados estatístico o C. maculatus, quando a aplicação foi feita com uma dosagem de $12 \mathrm{~mL}$.

Como se observa, o controle dessa praga se deve aos extratos empregados cujo efeito se manifesta pelos gases liberados, similar ao que ocorre com a tecnologia de controle de insetos-praga de grãos e sementes armazenados com produtos químicos, como a fosfina $\left(\mathrm{PH}_{3}\right)$, que se dá pela liberação dos gases quando da decomposição do produto comercial. 

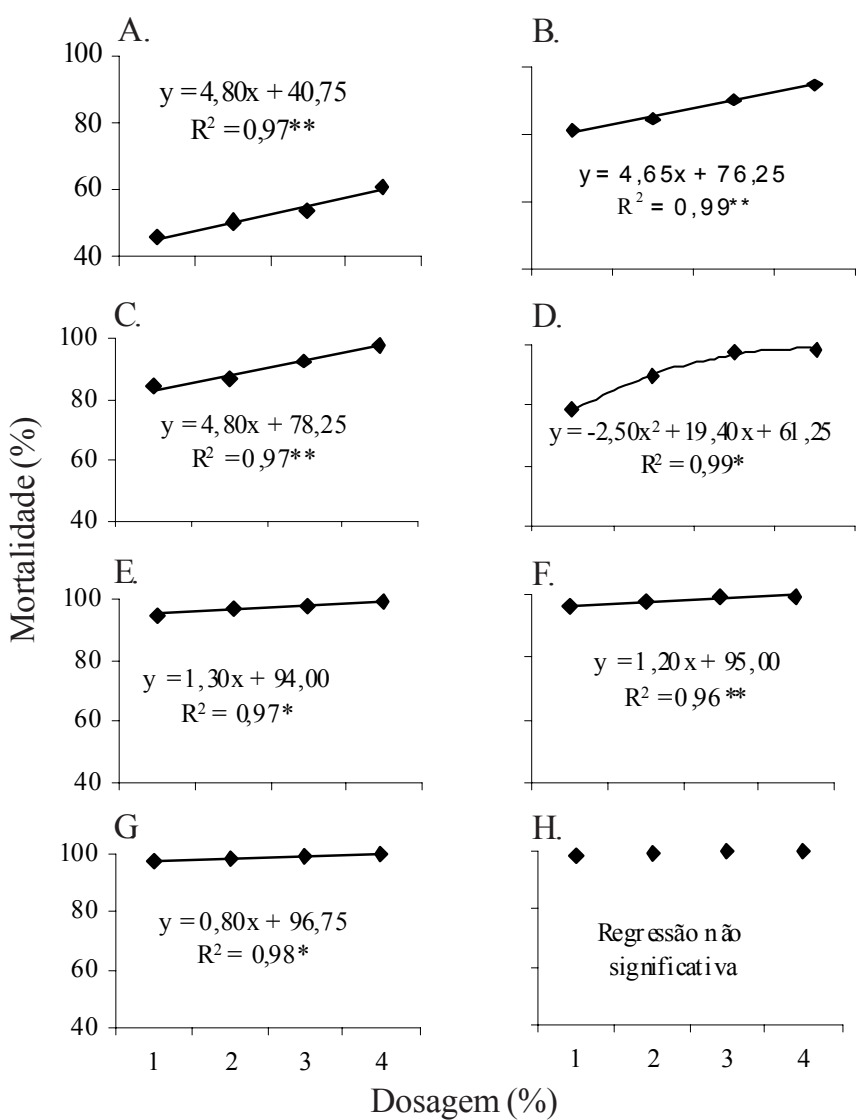

Figura 1. Mortalidade de Callosobruchus maculatus em função da dose aplicada do extrato de Camellia sinensis (A) Anthemis nobilis (B), Mentha piperita (C), Croton tiglium (D), Annona squamosa (E), Piper nigrum (F), Calopogonium caeruleum (G) e Azadiracta indica $(\mathrm{H})$

Tabela 3. Valores Médios da mortalidade (\%) de Callosobruchus maculatus para a interação extratos $\mathrm{x}$ dosagem pelo método de vapor após $48 \mathrm{~h}$ da aplicação dos extratos

\begin{tabular}{lccccc}
\hline & \multicolumn{5}{c}{ Dose Aplicada (mL) } \\
\cline { 2 - 6 } Tratamentos & 3 & 6 & 9 & 12 & Médias \\
\hline C. sinensis & $46,00 \mathrm{Cc}$ & $50,50 \mathrm{cBC}$ & $53,50 \mathrm{~dB}$ & $61,00 \mathrm{bA}$ & 52,75 \\
A. nobilis & $81,50 \mathrm{bB}$ & $84,50 \mathrm{bC}$ & $90,50 \mathrm{cA}$ & $95,00 \mathrm{aA}$ & 87,87 \\
M. piperita & $84,00 \mathrm{bC}$ & $86,50 \mathrm{bC}$ & $92,50 \mathrm{bcB}$ & $98,00 \mathrm{aA}$ & 90,25 \\
C. tiglium & $78,50 \mathrm{bC}$ & $89,00 \mathrm{bB}$ & $98,00 \mathrm{abA}$ & $98,50 \mathrm{aA}$ & 91,00 \\
A.scamosa & $95,00 \mathrm{aA} 97,00 \mathrm{aA}$ & $98,00 \mathrm{aA}$ & $99,00 \mathrm{aA}$ & 97,25 \\
P. nigrum & $96,00 \mathrm{aA} 97,50 \mathrm{aA}$ & $99,00 \mathrm{aA}$ & $99,50 \mathrm{aA}$ & 98,00 \\
C. caeruleum & 97,50 aA $98,50 \mathrm{aA}$ & $99,00 \mathrm{aA}$ & $100,00 \mathrm{aA}$ & 98,75 \\
A. indica & 98,50 aA $99,50 \mathrm{aA}$ & $100,00 \mathrm{aA}$ & $100,00 \mathrm{aA}$ & 99,50 \\
Médias & 84,62 & 87,87 & 91,31 & 93,87 \\
\hline * Média seguida pela mesma letra minúscula na coluna (DMS =6,25) e maiúscula na linha (DMS \\
= 5,27) não diferem estatisticamente pelo teste de Tukey a 1\% de probabilidade
\end{tabular}

A eficiência do $A$. indica se deve ao seu principal ingrediente ativo, a "azadiractina"; trata-se de um triterpeno, mais especificamente um limonóide, que causa distúrbios fisiológicos, alterando o desenvolvimento e a funcionalidade de várias espécies de insetos-praga, principalmente devido à ação de repelência alimentar, inibidora do desenvolvimento e crescimento e da reprodução (Ascher, 1993; Valladares et al., 1997). Lale \& Abdulrahman (1999) trabalhando com óleo da semente de $A$. indica, comprovaram sua eficiência no controle de população de C. maculatus.

\section{Métodos de aplicação dos extratos}

A análise de variância revelou efeito significativo para todas as fontes de variações analisadas, (Tabela 4), observando-se diferenças estatísticas entre todas as formas de aplicação dos extratos, pelo método do vapor e da aplicação direta, no tratamento de sementes do feijão Vigna unguiculata tipo "rabo de tatu", em silos metálicos de tamanho reduzido, visando à mortalidade dos C. maculatus, exceto entre as formas de aplicação "com e sem tubo", que foram iguais entre si, quando o extrato utilizado foi a $A$. squamosa. De acordo com os dados obtidos nesta análise, tem-se que a aplicação direta dos extratos na massa de sementes foi a mais eficaz em relação às demais formas de aplicação, por ter proporcionado o maior índice de mortalidade dos insetos após $48 \mathrm{~h}$ do tratamento, com exceção do extrato de $P$. nigrum, que se mostrou mais eficiente quando aplicado pelo método de vapor, com auxílio de um tubo de PVC perfurado em toda a sua extensão e introduzido no centro da massa de semente, o qual teve, como objetivo, diminuir a dificuldade de penetração dos gases liberados pelos extratos na massa de sementes permitindo, assim, melhor difusão de suas moléculas.

Tabela 4. Quadrado médio (QM) das análises de variâncias referentes ao número de insetos vivos, infestação inicial (ovos) depois de $48 \mathrm{~h}$ do tratamento das sementes e infestação final (orifício de emergência) de C. maculatus, depois de 30 dias

\begin{tabular}{lcccc}
\hline \multirow{2}{*}{ F.V } & G.L & $\begin{array}{c}\text { Insetos } \\
\text { Vivos }\end{array}$ & $\begin{array}{c}\text { Infestação } \\
\text { Inicial }\end{array}$ & $\begin{array}{c}\text { Infestação } \\
\text { Final }\end{array}$ \\
\cline { 3 - 5 } & & \multicolumn{3}{c}{ QM } \\
\hline Método de & 2 & $1730,51^{* *}$ & $3062,57^{* *}$ & $888,14^{* *}$ \\
Aplicação (M) & & $1694,70^{* *}$ & $3286,96^{* *}$ & $5449,91^{* *}$ \\
Extrato (E) & 4 & $16,86^{* *}$ & $168,91^{* *}$ \\
M x E & 8 & $216,87^{* *}$ & $412,86^{*}$ & 16,60 \\
Resíduo & 45 & 8,34 & 34,45 & 12,72 \\
CV(\%) & & 11,63 & 14,61 & \\
\hline
\end{tabular}

** Significativo a $1 \%$ de probabilidade

De forma geral e quando comparadas com a testemunha, todas as formas de aplicação utilizadas se mostraram eficazes pelos efeitos observados na redução do número de insetos vivos; entretanto, em termos de valores médios totais o método que melhor se destacou foi o da aplicação direta dos extratos no tratamento das sementes, e os extratos que obtiveram melhor desempenho na mortalidade dos C. maculatus foram os $C$. caeruleum e P. nigrum. Goldfarb (1997) utilizando extratos vegetais no controle do Sitophilus spp incoculados em uma massa de sementes de Zea mays BR 122, comprovou a eficiência do $P$. nigrum com uma mortalidade de $94 \%$ desses insetos. Cunha (2002) quando trabalhou com diferentes produtos vegetais no controle do C. maculatus, concluiu que o extrato de Bixa orellana foi o que proporcionou maior mortalidade do caruncho do feijão.

Em análise ao percentual de infestação inicial e final (Tabela 5) nota-se que a maior infestação ocorreu nas sementes não 
Tabela 5. Número de insetos vivos (A), infestação inicial (B) e infestação final (C) decorrentes da aplicação de $3 \mathrm{~mL}$ dos extratos no tratamento de sementes de feijão Vigna unguiculata var. "rabo de tatu"

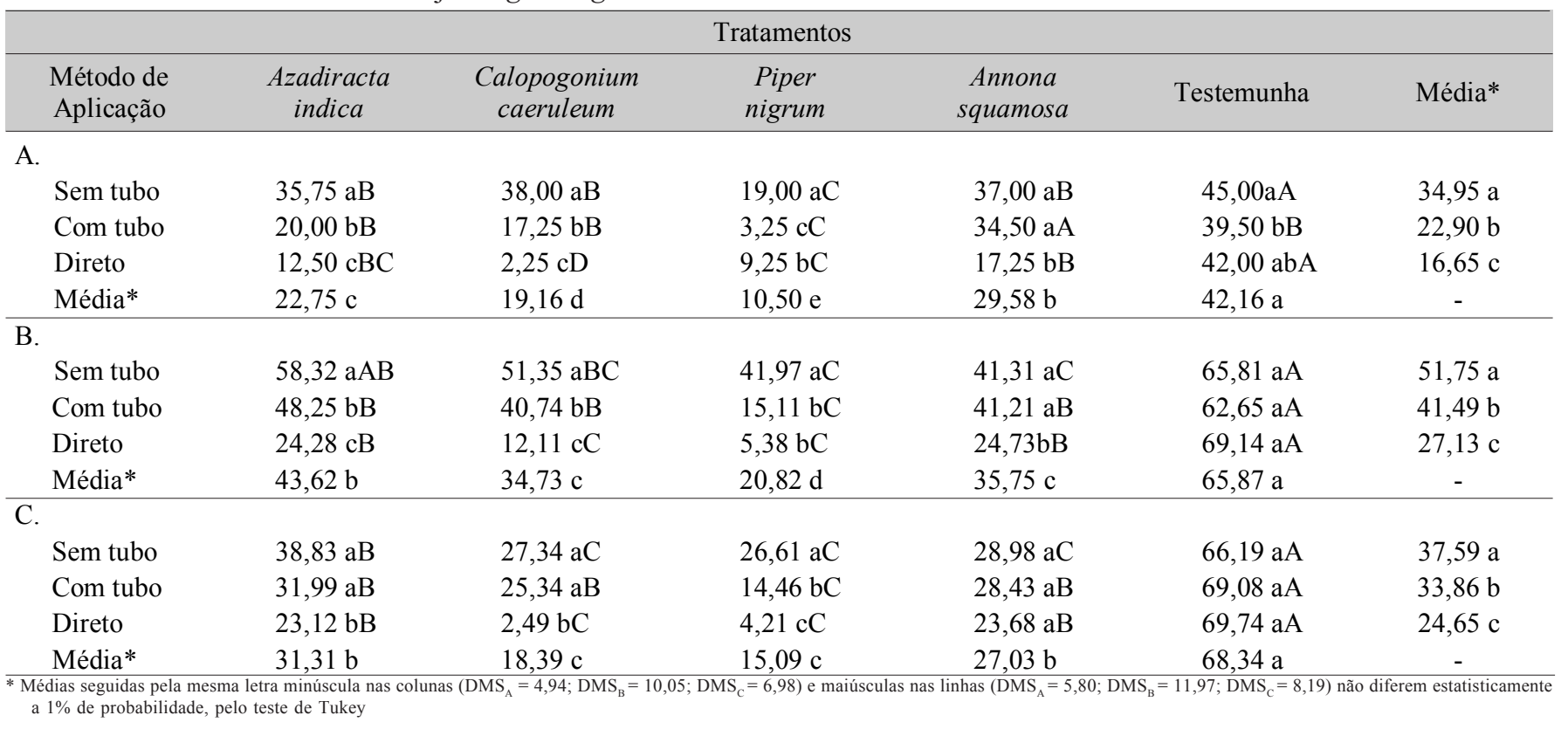

tratadas (testemunha) e que, mais uma vez, a aplicação direta, proporcionou os melhores resultados, ou seja, este método se sobressaiu dentre os demais, com exceção da forma com tubo, quando o extrato aplicado foi o P. nigrum (infestação inicial), e das formas "sem e com tubo", quando o extrato aplicado foi a A. squamosa (infestação final), em que não houve diferenças. Pode-se comprovar, ainda nesta tabela e com relação aos extratos empregados no tratamento das sementes, a eficiência do C. caeruleum e do P. nigrum, por terem inibido o desempenho de postura dos C. maculatus induzido, assim, um número menor de ovos postos sobre as sementes de feijão e, conseqüentemente, menor número de insetos emergidos. Este fato sugere que esses dois extratos são detentores de substâncias bioativas que, possivelmente, agem no aparelho respiratório desses carunchos, matando-os asfixiados, bem como interferindo na oviposição e viabilidade dos ovos. A utilização do extrato de $P$. nigrum n-hexânico 1000 ppm no tratamento de sementes de Zea mays, controlou significativamente a emergência do Sitophilus spp (Oliveira, 1998). Flores et al. (1993) sugeriram, quando trabalharam com extratos de P. nigrum no controle do caruncho Zabrotes subfasciatus em sementes de Phaseolus vulgaris armazenados, que a "piperina", principal ingrediente ativo desta espécie contra insetos-praga, poderia ser o responsável pelo relativo controle desses carunchos até 90 dias de armazenamento, quando aplicado em concentrações de 200 e 900 ppm.

\section{Inviabilidade de ovos de Callosobruchus maculatus}

A análise de variância da porcentagem de infestação revelada pelos orifícios de emergência provocados pelo $C$. maculatus ao final de 45 dias, apresentou efeito significativo a $1 \%$ de probabilidade para os fatores estudados e suas interações (Tabela 6).

Em análise aos dados contidos na Tabela 7, nota-se que o extrato de $P$. nigrum seguido do extrato de $C$. caeruelum utilizados no tratamento das sementes nas dose de 3 e $6 \mathrm{~mL}$,
Tabela 6. Quadrado médio da análise de variância referente à infestação final (orifício de emergência) de C. maculatus, depois de 45 dias

\begin{tabular}{lrr}
\hline \multirow{2}{*}{ Fonte de Variação } & \multicolumn{2}{c}{ Infestação Final } \\
\cline { 2 - 3 } & G.L & \multicolumn{1}{c}{ Q.M } \\
\hline Extratos (Ex) & 2 & $4796,36^{* *}$ \\
Dosagem (D) & 1 & $845,15^{* *}$ \\
Ex x D & 2 & $231,15^{* *}$ \\
Resíduo & 18 & \\
CV $(\%)$ & & 3,53 \\
** Significativo a 1\% de probabilidade & &
\end{tabular}

Tabela 7. Valores médios da infestação final (\%) de Callosobruchus maculatus para a interação tratamento $\mathrm{x}$ dose aos 45 dias de aplicação dos extratos pelo método direto

\begin{tabular}{lccc}
\hline \multirow{2}{*}{ Extratos } & \multicolumn{3}{c}{ Dose $(\mathrm{mL})$} \\
\cline { 2 - 4 } & 3 & 6 & Média* \\
\hline C. caeruleum & $64,83 \mathrm{bA}$ & $43,87 \mathrm{bB}$ & $54,35 \mathrm{~b}$ \\
P. nigrum & $52,57 \mathrm{cA}$ & $37,92 \mathrm{cB}$ & $45,25 \mathrm{c}$ \\
Testemunha & $91,47 \mathrm{aA}$ & $91,47 \mathrm{aA}$ & $91,47 \mathrm{a}$ \\
Média* & $69,62 \mathrm{~A}$ & $57,75 \mathrm{~B}$ & \\
\hline * Médias seguidas pela mesma letra minúscula na coluna (DMS $=4,0696)$ e maíscula na linha (DMS
\end{tabular}

$=3,3481$ ) não diferem estatisticamente pelo teste de Tukey a de $1 \%$ de probabilidade

apresentou-se eficiente quando comparado com a testemunha, pelos efeitos observados na redução do percentual de sementes com orifícios de emergência dos insetos comprovando, deste modo, seus efeitos ovicida. É oportuno notar que, além do efeito ovicida, esses extratos interferem no período de incubação dos ovos viáveis, aumentando o ciclo evolutivo dos insetos, fato este comprovado pela presença dos carunchos emergidos a partir dos 35 dias do tratamento das sementes ovipositadas, quando a dosagem aplicada foi de $6 \mathrm{~mL}$, enquanto na dosagem de $3 \mathrm{~mL}$, os carunchos surgiram a partir dos 30 dias; esses extratos apresentam, sem dúvida, constituintes químicos ativos 
que, possivelmente se difundem nos ovos dos C. maculatus, afetando o processo fisiológico e bioquímico da formação embrionária, evitando a viabilidade dos mesmos ou retardando o seu desenvolvimento. Constata-se, ainda, que a inviabilidade dos ovos aumenta com o aumento da quantidade da dose de extrato aplicado. Resultados semelhantes foram encontrados por Boff \& Almeida (1996) quando concluíram que todos os extratos de $P$. nigrum utilizados em seu trabalho, apresentaram efeito tóxico crescente para ovos de Sitotroga cerealella. Segundo Miyakado et al. (1989) os frutos de P. nigrum possuem alcalóides, especificamente do grupo de amidas insaturadas, com ação tóxica sobre muitas espécies de insetos-praga. $\mathrm{O}$ efeito ovicida para coleópteros da família Bruchidae já é bem esclarecido, em virtude dos estudos das estruturas dos ovos desses insetos. Desta forma, Credland (1992) estudou a estrutura dos ovos de quatro espécies de Callosobruchus e de Z. subfasciatus, a fim de explicar o eficiente efeito ovicida de óleos. Verificou-se, daí, que ovos de Callosobruchus têm um espaço largo incluso entre o ovo e o tegumento da semente. Este espaço é conectado com o exterior, através de um pequeno "funil" no final da parte posterior do ovo que, supostamente, é a razão para um bom efeito do ovicida e, sem dúvidas, também larvicida.

\section{CONCLUSÕES}

1. A mortalidade dos insetos está relacionada com o aumento da quantidade de extrato aplicado.

2. A aplicação dos extratos diretamente sobre a massa de sementes foi o método mais eficiente no controle ao Callosobruchus maculatus.

3. Os extratos de Calopogonium caeruleum e Piper nigrum, na dose de 3 e $6 \mathrm{~mL}$, mostraram-se eficientes com efeitos ovicidas.

\section{LITERATURA CITADA}

Almeida, F.A.C.; Goldfarb, A.C.; Gouveia, J.P.G. de. Avaliação de extratos vegetais e métodos de aplicação no controle de Sitophilus spp. Revista Brasileira de Produtos Agroindustriais, Campina Grande, v.1, n.1, p.13-20, 1999.

Ascher, K.R.S. Nonconventional insecticidal effects of pesticide available from neem tree (Azadirachta indica). Archives Insect Biochemistry and Physiology, Israel, v.22, n.3/4, p.433-449. 1993.

Boff, M.I.C.; Almeida, A.A. Ação tóxica de pimenta do reino, Piper nigrum, em ovos de Sitotroga cerealella (Lepidoptera: Gelechiidae). In: Congresso Brasileiro da Sociedade Entomológica do Brasil, 25, Londrina, 1996. Anais ... Londrina: SEB, 1996, p.423-429.

Credland, P.F. The structure of bruchid eggs may explain the ovicidal effect of oils. Journal of Stored Products Research, Canberra, v.28, n.1, p.1-9, 1992.

Cunha, E.M. Efeito de produtos vegetais e da fosfina no controle do Callosobruchus maculatus e na qualidade fisiológica de sementes de caupi (Vigna unguiculata). Areia: UFPB, 2002, 37p. Dissertação Mestrado
Flores, W.L.; Sampaio, L.S. de V.; Marques, O.M.; Costa, J.A. Efeitos dos extratos de pimenta do reino e cinamomo e do malation no controle do caruncho Zabrotes subfasciatus em sementes de feijão (Phaseolus vulgaris) armazenadas. Insecta, Salvador, v.2, n.1, p.11-22, 1993.

Goldfarb, A.C. Controle do inseto Sitophilus spp com extratos naturais de origem vegetal e seus efeitos na qualidade fisiológica em sementes de milho. Campina Grande: UFPB, 1997, 77p. Dissertação Mestrado

Hernández, C.R.; Vendramim, J.D. Avaliação da bioatividade de extratos aquoso de meliaceae sobre Spodoptera frugiperda. Revista de Agricultura, Piracicaba, v.72, n.3, p.305-317, 1997.

Kéita, S.M.; Vicent, C.; Schmit, J.; Arnason, J.T.; Bélanger, A. Efficacy of essential oil of Ocimum basilicul and O. gratissimum applied as na insecticidal fumigant and powder to control Callosobruchus maculatus. Journal of Stored Products Research, Austrália, v.37, n.4, p.339-349, 2001. http:/ /www.elsevier.com/locate/jspr. 22 fev. 03.

Lale, N.E.S.; Abdulrahman, H.T. Evaluation of neem (Azad ed isobutylamides: from natural products to agrochemical leads. In: Arnason, J.T.; Philogene, B.J.R.; Morand, P. (eds.), Insecticides of plant origin. Washington: American Chemical Society, 1999.213p.

Martinazzo, A.P.; Faroni, L.R.D.; Berbert, P.A.; Reis, F.P. Utilização da fosfina em combinação com o dióxido de carbono no controle do Rhyzopertha dominica (f.). Pesquisa Agropecuária Brasileira, Brasília, v.35, n.6, p.1063-1069, 2000.

Miyakado, M.; Nakayama, I.C.; Ohno, N. Insecticidal unsaturated isobutylamides: from natural products to agrochemical leads. In: Arnason, J.T.; Philogene, B.J.R.; Morand, P. (eds.), Insecticides of plant origin. Washington: American Chemical Society, 1989.213p.

Oliveira, M.M. Análise da eficácia de extratos vegetais no controle da praga, Sitophilus spp em grãos armazenado. Campina Grande: UFPB, 1998. 87p. Dissertação Mestrado

Prista, L.N.; Alves, A.C.M. Tecnologia farmacêutica. 4.ed. Lisboa: Fundação Calouste Guldenkian, 1996, p.1065-1078.

Silva, F.A.S. e; Azevêdo, C.A.V de. Versão do programa computacional Assistat para o sistema operacional windows. Revista brasileira de Produtos Agroindústriais, C. Grande, PB, v.4, n.1, p.71-78, 2002.

Tavares, M.A.G.C. Bioatividade da erva-de-santa-maria, Chenopodium ambrosioides L. (Chenopodiaceae), em relação a Sitophilus zeamais (COL.: Curculionidae). Piracicaba: ESALQ, 2002. 59p. Dissertação Mestrado

Valladares, G.; Defago, M.T.; Palacios, S. Laboratory evaluation of Melia azedarach (Meliaceae) extracts against the Elm Leaf Beetle Coleoptera: Chrysomelidae). Journal of Economic Entomology, Califórnia, v.90, n.3, 1997, p.747-750.

Vendramim, J.D. Plantas inseticidas e controle de pragas. Informativo da Sociedade Entomológica do Brasil, Piracicaba, v.25, n.2, 2000, p.1-5. 